\title{
Acquisition of Eyeblink Conditioning Is Critically Dependent on Normal Function in Cerebellar Cortical Lobule HVI
}

\author{
Philip J. E. Attwell, Shbana Rahman, and Christopher H. Yeo \\ Department of Anatomy and Developmental Biology, University College London, London WC1E 6BT, United Kingdom
}

\begin{abstract}
Classical conditioning of the nictitating membrane response (NMR)/eyeblink response of rabbits is a simple form of cerebellar-dependent, associative motor learning. Reversible inactivations of the cerebellar nuclei and inferior olive have implicated the olivo-cortico-nuclear loop in the acquisition of nictitating membrane conditioning, but the role of the cerebellar cortex in acquisition has not been tested directly. Here we have used local infusions of the water-soluble, disodium salt of 6-cyano-7-nitroquinoxaline-2,3-dione reversibly to block cerebellar cortical AMPA/kainate receptors in lobule HVI during acquisition training. After the drug effects dissipated, there was no evidence that acquisition had taken place; the subjects behaved as if naive. Further training without inactivation then allowed normal acquisition, and further inactivations during performance of conditioned responses abolished these estab-
\end{abstract}

lished responses. There was a strong correlation between the inactivation effects on acquisition and subsequent inactivation effects on performance, indicating that the same eyeblinkcontrol cortical microzones are engaged in learning and expressing this behavior. The cortical component of the olivocortico-nuclear loop is essential for acquisition of classically conditioned nictitating membrane response learning, and eyeblink control areas in $\mathrm{HVI}$ are critical. Our findings are consistent with models of cerebellar learning that assign essential plasticity to the cortex or to a distribution between levels in olivocortico-nuclear modules.

Key words: classical conditioning; acquisition; nictitating membrane; cerebellar cortex; AMPA receptors; reversible inactivation
The cerebellum is implicated in the acquisition and performance of motor skills, but whether motor memories are stored within its circuitry has not been resolved. Classical conditioning of the eyeblink and nictitating membrane response (NMR) has been useful for investigating motor learning mechanisms. NMR conditioning is known to be cerebellar dependent because lesions of discrete, eyeblink control regions of the cerebellar cortex, cerebellar nuclei, or inferior olive all impair performance of previously conditioned NMRs (for review, see Kim and Thompson, 1997; Yeo and Hesslow, 1998).

To analyze acquisition and storage processes for NMR conditioning, recent studies have used reversible inactivations of neural function during acquisition training. Localized infusions of the $\mathrm{GABA}_{\mathrm{A}}$ agonist muscimol during NMR conditioning trials, with subsequent testing after the drug effects have dissipated, revealed that normal activity in the cerebellar nuclei is critical for acquisition (Krupa et al., 1993; Hardiman et al., 1996; Yeo et al., 1997) and extinction (Hardiman et al., 1996; Ramnani and Yeo, 1996). Together with evidence that inactivations of cerebellar output in the superior cerebellar peduncle do not prevent acquisition (Krupa and Thompson, 1995), these findings provide strong support for the suggestion that essential plasticity for NMR conditioning is within the cerebellum.

Several theories suggest that a major component of motor learning is within the cerebellar cortex (Marr, 1969; Albus, 1971;

\footnotetext{
Received March 6, 2001; revised May 9, 2001; accepted May 18, 2001.

This study was supported by Biotechnology and Biological Sciences Research Council Grant 31/S10225. We thank Magnus Ivarsson for help with data and image analysis.

Correspondence should be addressed to Dr. Christopher H. Yeo, Department of Anatomy and Developmental Biology, University College London, London WC1E 6BT, UK. E-mail: c.yeo@ucl.ac.uk.

Copyright (C) 2001 Society for Neuroscience 0270-6474/01/215715-08\$15.00/0
}

Gilbert, 1974, 1975; Ito, 1982, 1998), and we have shown that there is convergence of information essential for NMR conditioning in cerebellar cortical lobule HVI (Yeo et al., 1985b), which contains the major eyeblink control regions (Hesslow, 1994a,b). However, if NMR conditioning is mainly mediated by cortical plasticity, why should inactivation of the cerebellar nuclei completely prevent it? We previously suggested one possibility (Ramnani and Yeo, 1996; Yeo et al., 1997). In addition to its effects on the excitatory output neurons of the cerebellar nuclei, muscimol will also deeply inhibit the inhibitory neurons that provide regulatory feedback to the inferior olive (Andersson et al., 1988). So, nuclear muscimol treatments would disturb the olivo-corticonuclear loop and could impair acquisition wholly, or partially, by disturbance of cortical excitability mediated by changed climbing fiber input (Fig. 1), consistent with the finding that direct inactivation of the inferior olive also prevents acquisition of NMR conditioning (Welsh and Harvey, 1998).

Reversible inactivation of critical cortical regions directly could test whether acquisition is dependent on normal cortical activity. If acquisition were to be normal, cerebellar cortex could be critically involved in neither acquisition nor information storage for NMR conditioning. In contrast, impairment of acquisition would implicate the cortex in the acquisition process and would also be consistent with information storage in the cortex, either as a principal component of the memory (Yeo et al., 1985a,b; Yeo and Hesslow, 1998) or as part of a distribution between the cortex and nuclei (Raymond et al., 1996; Ohyama and Mauk, 2001). Here, we test for these possibilities using localized infusions of the non-NMDA ionotropic glutamate receptor antagonist 6-cyano-7-nitroquinoxaline-2,3-dione (CNQX) reversibly to block cerebellar cortical AMPA/kainate receptor function during acquisition training. 


\section{MATERIALS AND METHODS}

\section{Surgery}

Nineteen male Dutch belted Rabbits $(1.8-2.5 \mathrm{~kg})$ were implanted with a guide cannula directed toward the right cerebellar lobule HVI. Subjects were anesthetized using a fentanyl-fluanisone mixture $(0.1-5.0 \mathrm{mg} / \mathrm{kg}$, i.m.) with benzodiazepam $(0.5 \mathrm{mg} / \mathrm{kg}$, i.v. $)$. Mannitol $(20 \% \mathrm{w} / \mathrm{v}, 30 \mathrm{ml}$ over $30 \mathrm{~min}$, i.v) was given to facilitate exposure of the cerebellum. Then, anesthesia was by fluothane $(1-2 \%)$ in oxygen/nitrous oxide $(2: 1)$. A cranial opening exposed cerebellar cortex and a 26 gauge (ga), $11 \mathrm{~mm}$ stainless steel guide cannula was implanted into the right HVI and fixed with acrylic cement. A 33 ga dummy cannula fitted into the guide kept the implant sealed. Postoperative analgesia (buprenorphine, 100 $\mu \mathrm{g} \cdot \mathrm{kg}^{-1} \cdot \mathrm{d}^{-1}$, i.m.) and antibiotic cover (chloramphenicol, 30 $\mathrm{mg} \cdot \mathrm{kg}^{-1} \cdot \mathrm{d}^{-1}$, i.m.) were given for $3 \mathrm{~d}$. Subjects were kept on a $12 \mathrm{hr}$ day/night cycle with ad libitum access to food and water.

Seven to $13 \mathrm{~d}$ after cannula guide implantation, a nylon monofilament suture loop was placed in the right nictitating membrane (NM) under local anesthesia (proxymetacaine hydrochloride, $0.5 \% \mathrm{w} / \mathrm{v}$ ).

\section{Conditioning apparatus and stimuli}

Rabbits were trained using techniques similar to those first developed by Gormezano et al. (1962) and described in detail by Yeo and Hardiman (1992). Subjects were placed in a restraining stock and movement of the NM was recorded using an isotonic transducer (Gormezano and Gibbs, 1988) linked to the membrane by the monofilament loop.

Each subject was placed in a ventilated, sound-attenuating chamber facing a centrally mounted loudspeaker. The conditioned stimulus (CS) was a $1 \mathrm{kHz}$ sine wave tone of $410 \mathrm{msec}$ duration and an intensity of 87 dBA. Background noise produced by ventilation fans was $57 \mathrm{dBA}$. The unconditioned stimulus (US) was peri-orbital electrical stimulation. Each US was a $60 \mathrm{msec}$ train of three biphasic current pulses $(2 \mathrm{~mA})$. The interstimulus interval between the CS and US onsets on paired trials was $350 \mathrm{msec}$. The intertrial interval was randomly selected between 25 and $35 \mathrm{sec}$. Each session consisted of 50 trials, and so the mean session duration was $25 \mathrm{~min}$.

\section{Nictitating membrane response recording}

Output from the NM transducer was fed to a 12-bit analog-to-digital convertor (CED 1401). Baseline and within-trial NM excursions were recorded to produce conditioned response $(\mathrm{CR})$ frequency and latency measures.

$C R$ frequency. A CR was defined as an NMR within the CS-US interval with amplitude $\geq 0.5 \mathrm{~mm}$ and with onset latency $>35 \mathrm{msec}$ from CS onset (Hardiman and Yeo 1992). CR frequency (\% CR) was calculated for each block of nine paired trials throughout the conditioning sessions and for each complete session.

CR onset latencies. The onset latency of every CR (defined as above) was measured as the first displacement of $0.05 \mathrm{~mm}$ beyond baseline after CS onset. These latencies were measured on all CS-US paired and CS alone, unpaired trials.

\section{Drug infusions}

When cortical infusions were given (see below), the dummy cannula was removed, and a sterile, 33 ga infusion cannula was inserted through the guide to project $1.7 \mathrm{~mm}$ below the guide tip. Each subject then received an infusion of CNQX (disodium salt, Tocris; $3 \mathrm{~mm}, 2 \mu \mathrm{l}$ in PBS, pH 7.4) or vehicle (PBS, $2 \mu \mathrm{l}$ ) over 2 min into the right cerebellar cortex.

\section{Experimental design}

A previous study (Attwell et al., 1999) and pilot experiments revealed that intracortical CNQX infusions can block cerebellar function and performance of all previously acquired CRs for $35 \mathrm{~min}$. Therefore, in the present study all behavioral sessions were set at 25 min (containing 50 trials) so that they were within the expected duration of the drug effects.

Habituation phase. After placement of a suture in the nictitating membrane (see above), all subjects received a single adaptation session. They were placed in the restraining stock within the conditioning chamber, and the NM transducer was fitted. Each subject rested quietly in the conditioning chamber for $25 \mathrm{~min}$, which is equivalent to the duration of one conditioning session, and no stimuli were presented. Subjects were randomly assigned to one of two groups: a CNQX-treated group or a vehicle-treated group.

Experimental phase 1: acquisition training with cortical infusions. Subjects received four daily sessions of acquisition training that began $5 \mathrm{~min}$ after the completion of either CNQX or vehicle infusions in the right cerebellar cortex. Each session consisted of 50 trials and lasted $25 \mathrm{~min}$. In 45 trials the CS and US were paired, and in 5 trials the CS was presented alone with no US. The CS-alone was presented on every 10th trial. Effective inactivation would produce low levels of conditioned responses in the CNQX-treated groups during this phase.

Experimental phase 2A: acquisition training with no infusions. Three days after the end of Phase 1, subjects received four daily sessions of acquisition training. Each session consisted of 50 trials (45 paired trials and 5 unpaired CS trials) and lasted $25 \mathrm{~min}$. All protocols were as in phase 1 except that no CNQX or vehicle infusions were given. If CNQX treatment had impaired acquisition in phase 1 , then the absence of CRs at the beginning of phase $2 \mathrm{~A}$ would be evidence for the impaired acquisition in phase 1 . The development of conditioning in phase 2 would be evidence that the cannulation and drug infusions had produced no permanent damage to critical structures.

Experimental phase 2B: acquisition training with no infusions (continued). Three days after the end of phase $2 \mathrm{~A}$, all CNQX-treated subjects received a further four daily sessions of acquisition training with all protocols as in phase $2 \mathrm{~A}$. If drug infusions had completely prevented acquisition in phase 1, then CR frequencies for CNQX-treated subjects in phase $2 \mathrm{~B}$ would be similar to those for vehicle-treated subjects in phase $2 \mathrm{~A}$.

Experimental phase 3: performance testing for efficacy of cortical infusions. To test whether the CNQX infusions in phase 1 had been in appropriate locations and sufficient to fully inactivate the critical control regions, in phase 3 we tested their efficacy in blocking the performance of conditioned responses established previously in phases $2 \mathrm{~A}$ and $2 \mathrm{~B}$. The phase 3 session began with 20 trials (18 paired CS-US trials and 2 unpaired CS trials). CNQX (same dose and concentration as in phase 1) was then infused. After 2 min, the session continued with 50 trials (45 paired CS-US and 5 unpaired CS) as in the conditioning sessions of phase 1 . In most cases, a further block of 50 trials was given immediately to establish stable asymptotic levels. In this way, the effects of CNQX infusion were assessed for a time period equivalent to, or beyond, a full training session in phase 1 .

\section{Histology}

In the final stage of the experiment, infusion cannulas were reinserted, and ${ }^{3} \mathrm{H}-\mathrm{CNQX}$ in PBS ( $3 \mathrm{~mm}$, containing $1 \mu \mathrm{Ci} / \mu \mathrm{l}, 2 \mu \mathrm{l}$ over $2 \mathrm{~min}$ ) was infused. This dose corresponds to that in phases 1 and $3 .{ }^{3} \mathrm{H}-\mathrm{CNQX}$ was infused in all subjects, including those from the PBS control group, so that the equivalence of cannula position and fluid delivery could be compared in the control and experimental subjects. Each subject was then given heparin sodium $(500 \mathrm{U} / \mathrm{kg}$, i.v. $)$ and an overdose of pentobarbitone sodium $(90 \mathrm{mg} / \mathrm{kg}$, i.v.) $17 \mathrm{~min}$ after the end of the CNQX inf usion. This time was chosen with reference to previous time course data (Attwell et al., 1999), and it corresponds to a time point in the middle of a training session. Each subject was perfused transcardially with $0.9 \%$ saline (1 liter) followed by $4 \%$ formaldehyde solution ( 2 liters). The brain was removed, embedded in gelatin, and cryoprotected in $20 \%$ sucrose solution, and then serial, $50 \mu \mathrm{m}$ frozen transverse sections were cut.

\section{Autoradiography and image analysis}

Every sixth brain section was opposed to tritium-sensitive film (Hyperfilm, Amersham) for autoradiography together with tritium standards (Microscales, Amersham) for 6 weeks at $4^{\circ} \mathrm{C}$. After film development, the sections were stained with cresyl violet. The autoradiograph of every brain section was imaged with a monochrome charge-coupled device camera and analyzed using standard densitometry techniques (M5+, Imaging Research Inc.,); the resultant images were calibrated, and their densities were color coded with reference to the tritium standards as picomoles of CNQX per milligram of tissue equivalent.

The stained sections were realigned to allow registration of the histology and autoradiography. An image of each Nissl-stained section was captured and processed to reveal the brain edges and granule cell layer boundaries. Composites of the color-coded densitometry and the brain contours were then made.

The Nissl-stained sections were examined for signs of cannula-induced damage in critical cerebellar regions. In particular, the more ventral regions of lobule HVI and the cerebellar nuclei were studied. Subjects with damage to these regions were excluded from the study. 

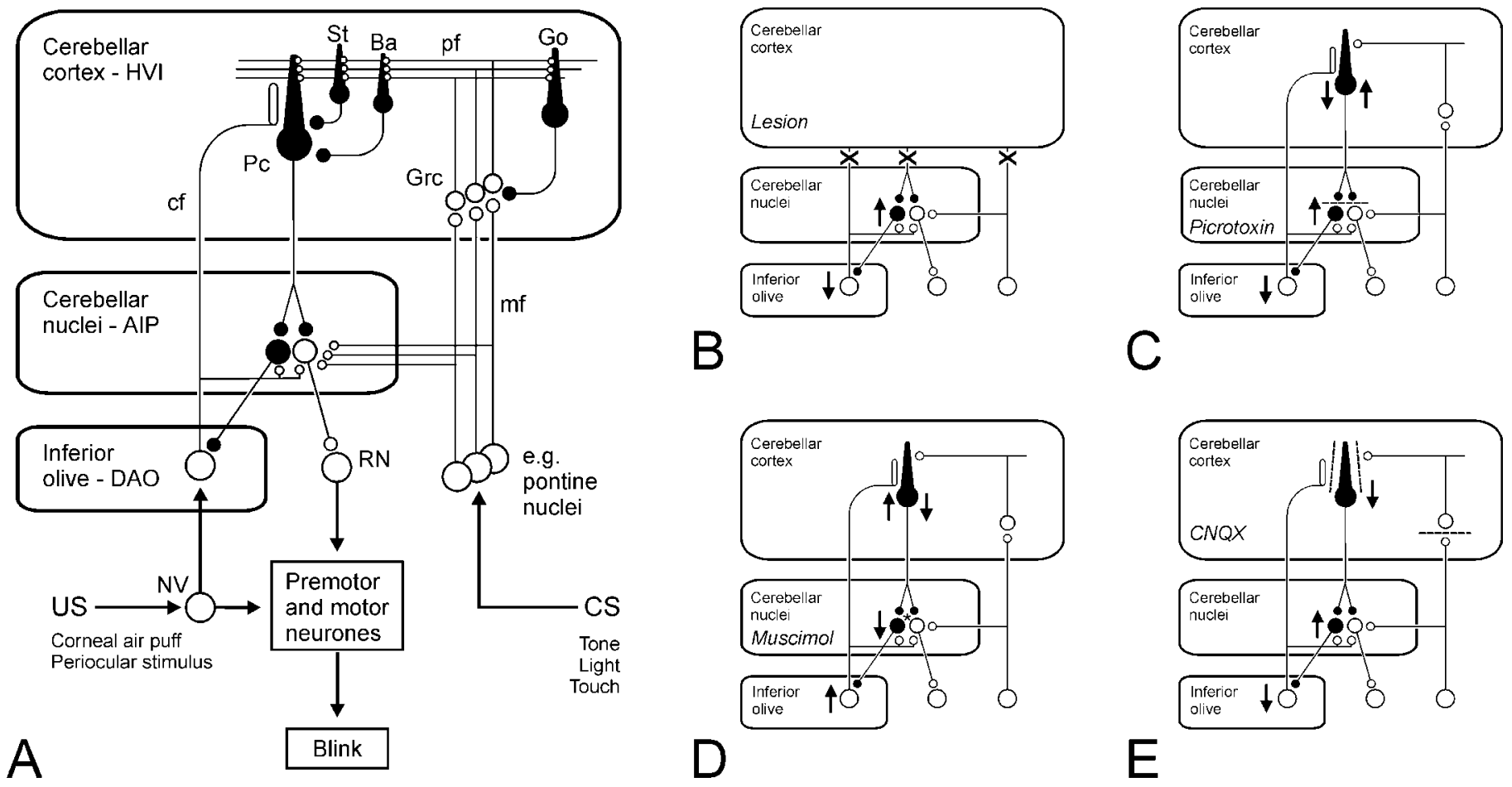

Figure 1. Olivo-cortico-nuclear loops: lesions and inactivations. A, A model of the cerebellum as a mediator of eyeblink conditioning. CS- and US-related information converges within the cerebellar cortex and within the cerebellar nuclei through mossy fiber and climbing fiber inputs, respectively (for review, see Yeo and Hesslow, 1998). Excitatory neurons and synapses are shown in white; inhibitory neurons and synapses are shown in black. AIP, Anterior interpositus nucleus; $B a$, basket cell; $c f$, climbing fiber; $D A O$, dorsal accessory olive; Go, Golgi cell; Grc, granule cell; $H V I$, cortical lobule HV I; $N V$, trigeminal nucleus; $P c$, Purkinje cell; $p f$, parallel fibers; $R N$, red nucleus; $S t$, stellate cell. $B-E$, Simplified views of the circuitry shown in $A$, with cortical interneurons, multiple mossy fiber inputs, and some brainstem circuits omitted for clarity. Each panel shows how information transmission and excitabilities within the olivo-cortico-nuclear loop may change after a different intervention. Excitability increases $(\uparrow)$ and decreases $(\downarrow)$ are indicated. $B$, After a cortical lesion, loss of Purkinje cell inhibition leads to increased excitabilities in the cerebellar nuclei and their efferent targets, consistent with enhanced unconditioned reflex eyeblinks (Yeo and Hardiman, 1992; Gruart and Yeo, 1995). In previously conditioned subjects, this lesion abolishes conditioned NM responses (Yeo et al., 1985a; Yeo and Hardiman, 1992) but can unmask short-latency, CS-driven eyelid responses (Perrett et al., 1993), consistent with the suggestion that conditioning induces plasticity within the cerebellar nuclei. $C$, Blockade of $\mathrm{GABA}_{\mathrm{A}}$ receptors in the cerebellar nuclei (indicated by barred synapses) by local picrotoxin infusions disinhibits excitatory output neurons and the inhibitory nucleo-olivary projection. Increased inhibition in the inferior olive has effects similar to cutting or reversibly cooling climbing fiber inputs to the cortex (Colin et al., 1980; Montarolo et al., 1982) to produce a loss of complex spikes but a significant increase in Purkinje cell, simple spike frequencies. This blockade can also unmask short-latency, CS-driven eyelid responses (Garcia and Mauk, 1998). D, Muscimol infusions in the cerebellar nuclei (active at synapses marked with an asterisk) agonize $\mathrm{GABA}_{\mathrm{A}}$ receptors and strongly depress nuclear excitabilities. Nucleo-olivary inhibition is depressed so olivary excitability will be increased. Increased climbing fiber activity increases complex spike activity with a corollary reduction in simple spike activity (Andersson and Hesslow 1987); this dual excitability change is indicated by $\uparrow$ and $\downarrow$. Nuclear muscimol infusions prevent acquisition and extinction of NMR conditioning (Krupa et al., 1993; Hardiman et al., 1996; Ramnani and Yeo, 1996; Yeo et al., 1997). The disruption of acquisition can relate to loss of normal function at any level in the olivo-cortico-nuclear loop. E, CNQX infusions in the cerebellar cortex block ionotropic, non-NMDA receptor-mediated transmission. The main targets (shown as barred synapses) are parallel fiber inputs to Purkinje cells (and cortical interneurons; data not shown), climbing fiber inputs to Purkinje cells, and mossy fiber to granule cell synapses. The block of parallel fiber synapses would reduce simple spike activity in Purkinje cells but may not abolish spontaneous activity. Cerebellar nuclear neurons would be partially disinhibited. Cortical CNQX infusions block performance of established NM conditioned responses (Attwell et al., 1999). In the present study, CNQX inf usions reveal no short-latency, CS-driven responses but they do prevent acquisition in naive subjects.

\section{Data analysis}

Where CR frequency and latency data passed tests for normality and homogeneity of variance, they were analyzed using a two-way, repeated measures ANOVA followed, where appropriate, by Newman-Keuls multiple comparisons test on the individual means. Data that failed normality or homogeneity of variance tests were analyzed using the Wilcoxon rank-sum test on main group effects over each phase.

\section{RESULTS}

\section{Assessment of infusion sites, infusion efficacies, and cannulation-related damage}

To effectively test cerebellar cortical function in NMR conditioning, the cannulation and infusions in each subject needed to satisfy three criteria. First, the CNQX infusions needed to be restricted to the cerebellar cortex. This criterion was tested by analysis of the ${ }^{3} \mathrm{H}-\mathrm{CNQX}$ autoradiography. Second, the CNQX infusions needed to have been effective in blocking function in the critical cerebellar cortical regions. This criterion was tested in phase 3. Only if CNQX fully blocked expression of conditioned responses for at least some part of the period after infusion was the subject included in the effective HVI-CNQX group. Finally, the cannulation should not have produced permanent damage sufficient to invalidate assessment of cortical function. To enable this control, the Nissl-stained sections were critically examined, and subjects with extensive, cannulation-related cortical damage in HVI or adjacent regions were excluded from further analysis.

The study began with 19 cannulated subjects. Ten were allocated to the CNQX group, and 9 were allocated to the PBS control group. At the end of the experiment, two subjects (one from the CNQX group and one from the PBS group) were found to have substantial cannulation-related damage in the cerebellar 


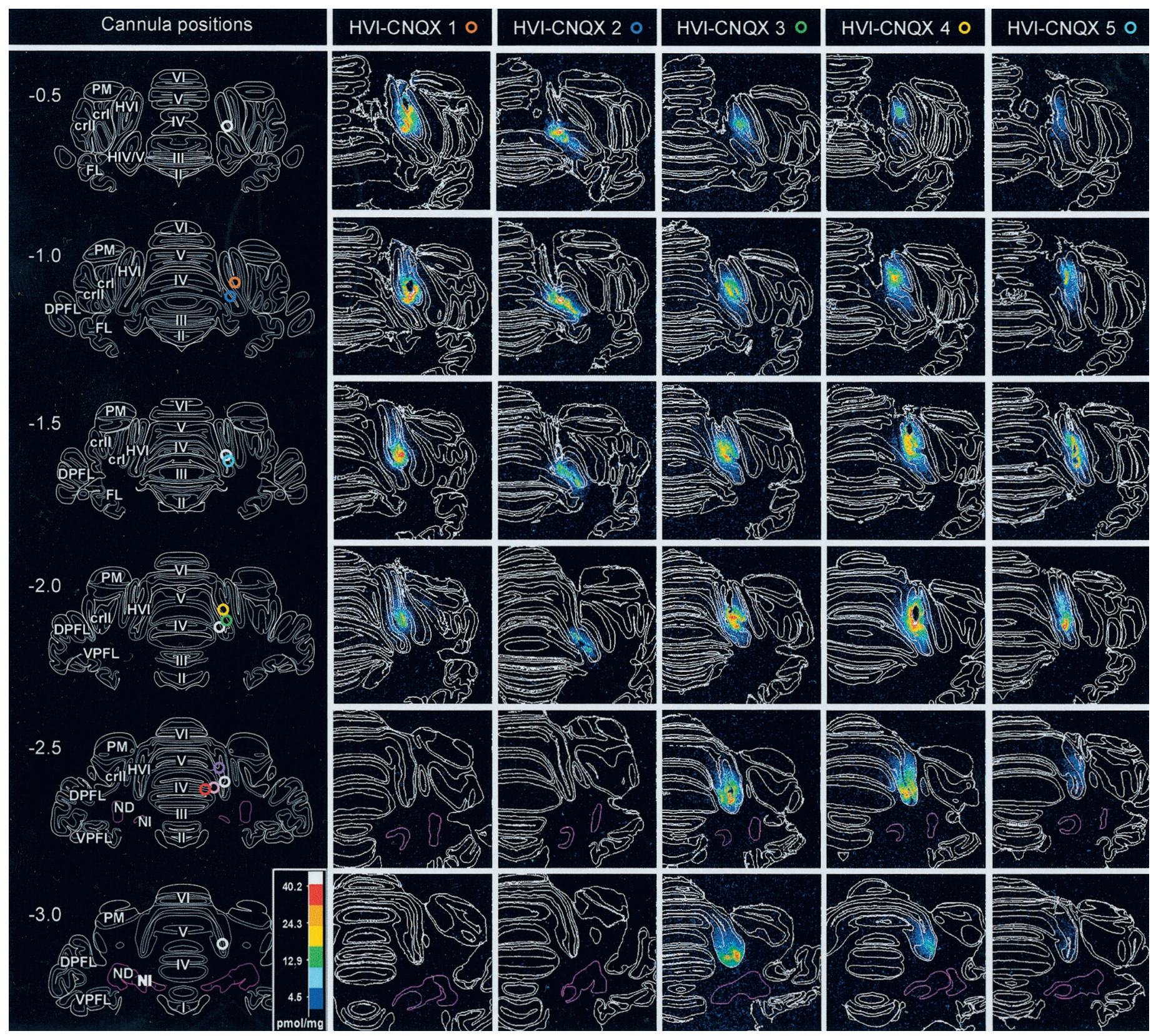

Figure 2. Cannula tip positions and ${ }^{3} \mathrm{H}-\mathrm{CNQX}$ distributions after localized inf usions into cerebellar cortex. In column 1, cannula tip locations are shown for all subjects on a series of six, standard transverse sections at levels from $0.5 \mathrm{~mm}$ anterior to $3.0 \mathrm{~mm}$ posterior to skull lambda. White rings indicate locations for the vehicle-infused subjects in the HVI-control group. Colored rings indicate locations for subjects in the HVI-CNQX and CTX-CNQX groups and are identified against each of these subjects in the autoradiography column headings. The cerebellar nuclei are shown with pink boundaries. Columns 2-6 and 7-9 show a series of actual transverse sections for all CNQX-infused subjects at levels corresponding to the standards. Subjects HVI-CNQX 1-5 are ranked by CNQX effects on behavior; HVI-CNQX 1 is most affected. Lobule and granule cell boundaries are shown in white. The density of ${ }^{3} \mathrm{H}-\mathrm{CNQX}$ binding is color-coded. Densitometry calibration: picomoles of CNQX per milligram tissue equivalent.

cortex. These two subjects were rejected from the study, and their data are not analyzed further.

Of the remaining nine CNQX group subjects, six were found to show a complete suppression of CRs in phase 3. In five of these, autoradiography of ${ }^{3} \mathrm{H}-\mathrm{CNQX}$ binding revealed that the infusions were confined to cortical lobule HVI. In one of these six, there was CNQX binding in the cortex but also in the cerebellar nuclei, so this subject was rejected. The five effective cortically confined infusions varied slightly in their spread in the rostralcaudal dimension, but in all cases, there was clear evidence of CNQX binding in the medial aspect of rostral HVI (Fig. 2,
HVI-CNQX 1-5). This location is consistent with the region identified in our earlier analysis of cortical regions engaged in CR performance (Attwell et al., 1999). There was no evidence of any CNQX binding in the cerebellar nuclei in these five subjects, so they form the HVI-CNQX group (Fig. 2). The three remaining subjects showed incomplete impairments of CR frequency during phase 3 of the experiment, suggesting that critical regions of the cerebellar cortex had not been infused, and autoradiography confirmed that CNQX binding was distributed differently in these subjects. In all cases the binding was more medial than that in the HVI-CNQX group subjects, and it included lobules IV and V of 


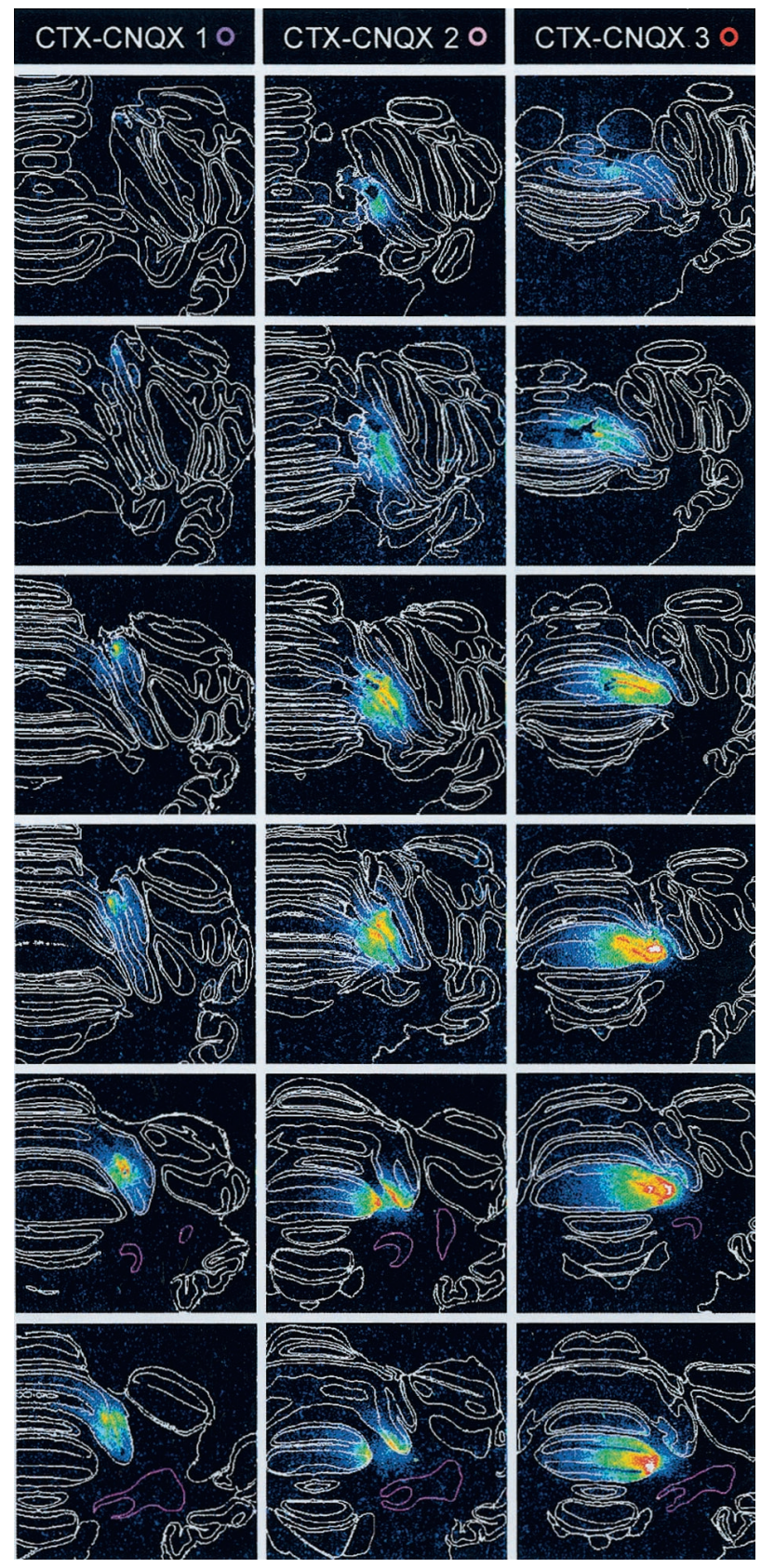

Figure 2. Continued

the anterior lobe. In one case (Fig. 2, CTX-CNQX 1), there was some binding in medial HVI, but it was at more caudal levels than in the HVI group, and so it appears to be mostly outside the previously identified critical region in HVI. Similarly, subject CTX-2 had some CNQX binding in medial HVI, but less than, for example, subject HVI-3 in the HVI-CNQX group. There was no evidence of CNQX binding in the cerebellar nuclei in these subjects, which form the CTX-CNQX subgroup.

Of the remaining eight PBS control group subjects, autoradiography (data not shown) revealed that in three subjects, the cannula tips and the majority of ${ }^{3} \mathrm{H}-\mathrm{CNQX}$ binding were in the inferior colliculus. These three subjects were excluded from the study. All of the final residual five PBS controls had cannula tip placements centered in HVI, and control CNQX binding was confined mainly to this lobule. These five subjects form the HVI-control group.

\section{Cerebellar cortical CNQX infusions impaired acquisition}

Subjects in the HVI-control group acquired CRs normally, reaching a stable level of learning (at least eight CRs in the nine paired presentations per block) in $166 \pm 22.5$ trials (mean \pm 1 SEM). Comparison of CR frequencies in the HVI-CNQX and the HVI-control group revealed significant overall main group differences during phase 1 (Wilcoxon rank-sum, $W=484.0 ; n_{1}=$ $\left.20, n_{2}=20 ; p<0.05\right)$. HVI-control subjects reached $63.2 \pm$ $18.9 \% \mathrm{CR}$ (mean \pm SEM) by session 4 , whereas the HVICNQX-treated subjects showed no responses (0\%) throughout this phase (Fig. 3). The complete absence of CRs through phase 1 in the HVI-CNQX subjects is consistent with the complete performance block that the drug can produce (Attwell et al., 1999), but at this stage it does not reveal whether acquisition was prevented. The test for whether there was acquisition during cortical inactivation in phase 1 is performance during session 5, at the start of phase $2 \mathrm{~A}$, when there was no inactivation. During this session, the control group produced $94.6 \pm 4.2 \% \mathrm{CR}$ (mean \pm 1 SEM), whereas the HVI-CNQX subjects only achieved $0.9 \pm$ $2.0 \% \mathrm{CR}$ (mean $\pm 1 \mathrm{SEM}$ ), indicating a substantial impairment of acquisition in phase 1 . For the entire phase $2 \mathrm{~A}$, main group comparisons reveal a highly significant difference between HVICNQX and HVI-control groups (Wilcoxon rank-sum, $W=$ 535.5; $\left.n_{1}=20, n_{2}=20 ; p<0.05\right)$; therefore, the cortical inactivation had produced a highly significant impairment of acquisition. This impairment was severe, because acquisition by HVI-control subjects during phase 1 did not differ significantly from that of HVI-CNQX subjects during phase 2A (RM ANOVA, $F=1.09 ; p>0.05)$. Thus, the HVI-CNQX subjects had acquired little or no CS-US association during the inactivation in phase 1 , and they started acquisition in phase $2 \mathrm{~A}$, as if naive. That they then acquired at a rate similar to naive control animals indicates that they recovered fully from the inactivation and that any general impairments attributable to cannulation were similar in the two groups.

The three subjects in the CTX-CNQX group showed clear evidence of acquisition during phase 1 and through phase $2 \mathrm{~A}$ (Fig. 3) consistent with testing in phase 3, which revealed that their cortical infusions did not fully block CR performance. Thus subjects with incomplete inactivation of the critical, eyeblink/NM control regions of lobule HVI were able to acquire conditioned responses relatively normally.

\section{Acquisition impairments correlate with efficacy of cortical AMPA/kainate receptor inactivations}

After the acquisition phases of the experiment, all subjects reached asymptotic performance, and then, during a training session in phase 3 , the effects of cortical CNQX on performance of existing CRs was assessed. Within and across the HVI-CNQX and CTX-CNQX group subjects there were differences in the severity of performance impairments (as measured in phase 3) and acquisition impairments (as measured in phase 2A). Previously, we have proposed that the severity of CR performance impairments reflects the completeness of the inactivation of the cortical eyeblink control areas (Attwell et al., 1999). Here we find that there is a strong correlation between the degree of performance block (as measured by the number of 10-trial blocks with $0 \% \mathrm{CR}$ in phase 3 ) and the rate of acquisition (as measured by the number of trials until responses reached $90 \%$ on paired trials within a block in phase $2 \mathrm{~A}$ ) (Spearmans rank correlation coefficient $=0.851 ; p<0.05$ ) (Fig. 4). This finding supports the 


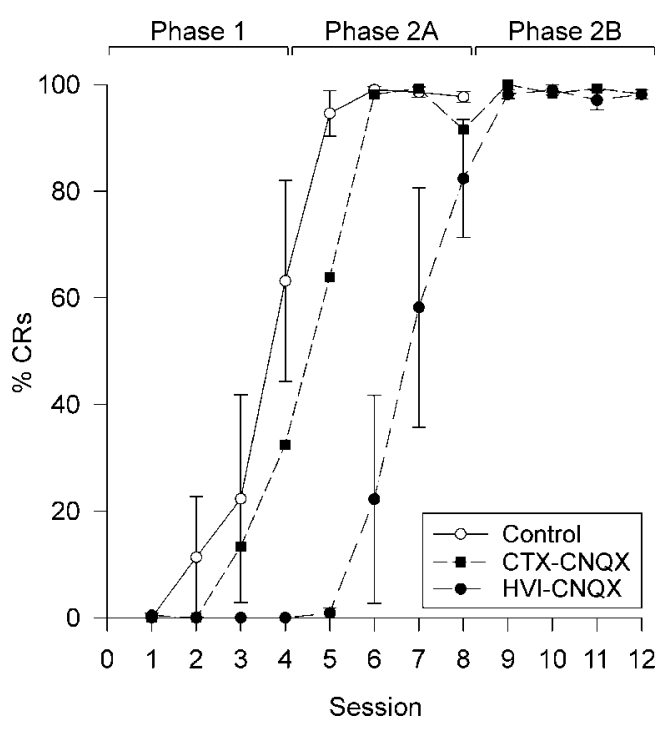

Figure 3. Effects of cerebellar cortical CNQX on acquisition of NMR conditioning. Daily, mean session \% CR ( \pm SEM) for control, CTXCNQX, and HVI-CNQX groups. Subjects in control and CTX-CNQX groups developed CRs during phase 1 and reached asymptotic CR frequencies in phase 2A. Subjects in the HVI-CNQX group showed no CRs in phase 1 and reached asymptotic CR frequencies in phase $2 \mathrm{~B}$.

suggestion that the same cortical regions involved in the performance of conditioned responses are those that actively engage in the acquisition process.

\section{CNQX infusions in lobule HVI abolish CRs or extend their latencies}

Recent studies have revealed short-latency, CS-driven eyelid responses in conditioned rabbits after anterior lobe lesions or blockade of GABAergic inhibition on the cerebellar nuclei by nuclear inf usions of picrotoxin (Garcia and Mauk, 1998). Here, we report the onset latencies of all CRs during the whole of phase 3 performance testing (Fig. 5). The main effect of CNQX infusions in HVI was to abolish CRs, so CR frequencies are significantly reduced, but as the drug effects dissipated, CRs gradually returned and their onset latencies were seen to be mildly extended in the HVI-CNQX group. Subjects in the CTX-CNQX group had infusions in anterior lobe regions adjacent to HVI, and these were largely ineffective in abolishing CRs. However, these subjects also showed extensions of CR onset latency that may relate to weak spread of the CNQX into HVI eyeblink control areas. There is no evidence, in either group, for CNQX-related reductions of CR onset latencies.

\section{DISCUSSION}

In an earlier study, we identified a region of the cerebellar cortex critical for the expression of conditioned NM/eyelid responses. Blockade of cerebellar cortical AMPA/kainate receptors using CNQX inf usions into a specific region of lobule HV I completely but reversibly blocked the production of previously established conditioned responses (Attwell et al., 1999). Now we have shown that in naïve subjects a similar blockade of glutamatergic transmission in this same cortical region prevents the acquisition of conditioning. So, normal function in the cerebellar cortical lobule HVI eyeblink control regions is essential for acquisition of eyeblink/NM classical conditioning. What are the implications of these findings for our understanding of where and how this motor learning is stored?

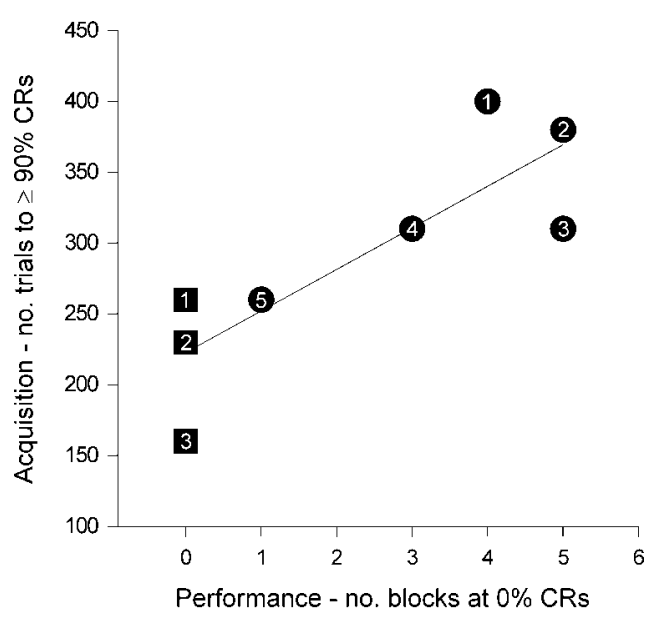

Figure 4. Correlation of CNQX effects on acquisition and subsequent performance. Acquisition and performance measures for subjects CTXCNQX 1-3 (each subject numbered in a square symbol) and HVICNQX 1-5 (each subject numbered in a circle symbol) groups. Subject identifiers correspond to those in Figure 2. $y$-axis: CNQX effects on acquisition (expressed as number of trials in phases 1,2A, and 2B needed to reach a criterion of $90 \% \mathrm{CR}$ within a 10 -trial block). $x$-axis: CNQX effects on performance (expressed as total number of 10-trial blocks in which CR frequency was $0 \%$, during the phase 3 performance testing session).

Because simple, delay eyeblink or NMR conditioning can be obtained in reduced, decerebrate preparations (Mauk and Thompson, 1987; Hesslow, 1995), it is clear that cerebellar and brainstem circuitry are sufficient to support its development and maintenance. Furthermore, inactivation of cerebellar output, in the superior cerebellar peduncle, does not impair acquisition (Krupa and Thompson, 1995), so we can be certain that memory storage for NMR conditioning is not further downstream, in the rubral, thalamic, or brainstem targets of cerebellar output. Instead, essential plasticity for eyeblink/NMR conditioning must be within the cerebellum itself, or the precerebellar brainstem circuitry, or both. The early success of cerebellar nuclear inactivations, using the $\mathrm{GABA}_{\mathrm{A}}$ agonist muscimol, in preventing acquisition (Krupa et al., 1993; Hardiman et al., 1996; Yeo et al., 1997) and extinction (Hardiman et al., 1996; Ramnani and Yeo, 1996) invited the suggestion that major plasticity for NMR conditioning could be within the cerebellar nuclei, but it is not clear how the relatively simple circuitry and synaptic organization of the nuclei might account for all of the complexities of context recognition and response timing that are critical features of learned motor skills, even those as apparently simple as the conditioned eyeblink.

An alternative interpretation of cerebellar nuclear inactivation effects is that they destabilize the olivo-cortico-nuclear loop and thereby have more general effects on cerebellar function by altering information processing at olivary, cortical, and nuclear levels (Ramnani and Yeo, 1996; Yeo et al., 1997). Muscimol infusions within the cerebellar nuclei affect two populations of Purkinje cell target neurons. One set is excitatory to rubral and thalamic targets, and the other set is inhibitory on inferior olive neurons (Andersson et al., 1988). Disturbance of this inhibitory, nucleo-olivary pathway leads to distinct changes in olivo-cortical transmission (Andersson and Hesslow, 1987), with further consequences for cortical and nuclear excitability changes (Colin et al., 1980; Montarolo et al., 1982). So, impairments of acquisition during muscimol (Krupa et al., 1993; Hardiman et al., 1996; Yeo et al., 1997) or lidocaine (Nordholm et al., 1993) inactivations of 

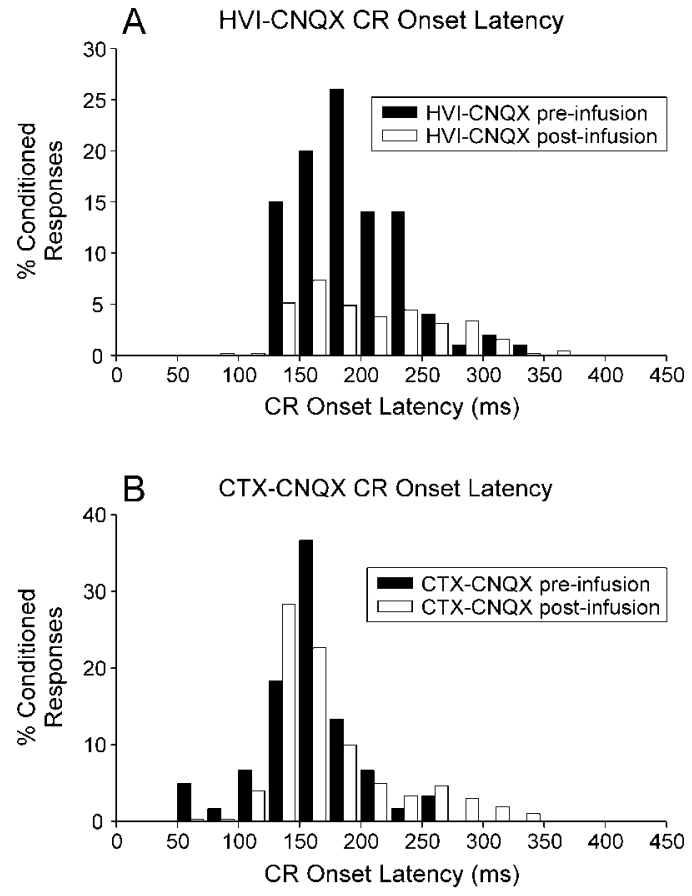

Figure 5. Distribution of $\mathrm{CR}$ onset latencies during administration of, and recovery from, cortical CNQX infusions. $A$, Frequency histogram (25 msec bin widths) of CR onset latencies for all subjects in the HVICNQX group before and after CNQX infusions. \% CRs are derived from 20 preinfusion trials (all subjects) and 50 (1 subject) or 100 (4 subjects) post-infusion trials. There is an overall reduction of CR frequency but little effect on the distribution of $\mathrm{CR}$ onset latencies. $B$, Frequency histogram ( $25 \mathrm{msec}$ bin widths) of CR onset latencies for all subjects in the HVI-CTX group before (20 trials) and after (100 trials) CNQX infusions. There is a mild extension of some CR onset latencies to between 250 and $350 \mathrm{msec}$ and no evidence of CR onset latency reductions.

the cerebellar nuclei do not directly confirm the locus of plasticity essential for conditioning. This plasticity may be at nuclear, olivary, or cortical levels or, indeed, distributed between them. Consistent with this idea is the finding that inactivation of the inferior olive also prevents acquisition of NMR conditioning (Welsh and Harvey, 1998) because olivary inactivation also profoundly changes cortical and nuclear activities (Colin et al., 1980; Montarolo et al., 1982). Furthermore, inactivations of cerebellar output, in the superior cerebellar peduncle, that do not prevent conditioning (Krupa and Thompson, 1995) were probably sufficiently distal to spare the nucleo-olivary inhibitory fibers, which exit the main body of the peduncle proximally and travel ventrally to the olive and so would not be expected to disturb olivary excitability through this direct route.

In the experiment reported here, inactivation of AMPA/kainate receptor-mediated transmission in the cerebellar cortex was also fully effective in preventing the acquisition of NMR conditioning, again consistent with the suggestion that normal function throughout the olivo-cortico-nuclear loop is necessary. If acquisition had not been affected by cortical disruption, then it would have been clear that there is no essential plasticity for NMR conditioning within the cerebellar cortex; however, because acquisition was prevented, we conclude that cortical, nuclear, and olivary plasticity are all candidate mechanisms in NMR conditioning.

Autoradiography confirmed that the region of cerebellar cortex critical for acquisition of NMR conditioning is within the medial part of rostral lobule HVI, a region that we identified previously as critical for performance of existing conditioned NM responses in lesion studies (Yeo et al., 1984, 1985a; Hardiman and Yeo, 1992; Yeo and Hardiman, 1992) and with reversible inactivations (Attwell et al., 1999). In cats and ferrets, eyeblink cortical microzones have been identified electrophysiologically within the $\mathrm{C} 1$ and C3 zones of lobule HVI (Hesslow, 1994a; Hesslow and Ivarsson, 1994). Those locations are highly consistent with these identified here as essential for acquisition of NMR conditioning in rabbits. In the present experiment, we retested the CNQX effects on the performance of established CRs in phase 3, after assessing its effects on acquisition in phases 1,2A, and 2B. We saw a strong correlation between the severity of acquisition and performance impairments produced by cortical inactivations in individual subjects, consistent with the view that this same cortical region is involved in both the acquisition and performance of learned responses and with the basic features of a cerebellar cortical conditioning model (Yeo and Hesslow, 1998). In this model, plasticity essential for NMR conditioning is assumed to develop at parallel fiber/Purkinje cell synapses within eyeblink control microzones. There is good evidence to support the suggestion that mossy fiber-parallel fiber pathways signal the CS in conditioning (Hesslow et al., 1999), although evidence supporting the role of the climbing fibers supplying US-related information as a reinforcing input is less clear (for review, see Yeo and Hesslow, 1998).

As in our earlier study of conditioned response performance, we have not systematically studied the possible engagement of other cerebellar cortical regions beyond lobule HVI because inactivations within this region were clearly sufficient to produce major impairments of acquisition and performance. However, electrophysiological mapping has revealed additional eyeblink control regions in HVII and paramedian lobes in cats, and one HVI eyeblink microzone may have an extension into lobule V (Hesslow, 1994b). We do not rule out the possibility that these other cerebellar cortical regions may also contribute to the development of NMR conditioning, but we have not explored them in any detail. It should be noted, however, that in two subjects (CTX-2 and CTX-3) there was clear binding of CNQX in lateral parts of lobules V and IV of the anterior lobe. Neither of these subjects had significantly impaired acquisition or performance of NMR conditioning, so our evidence suggests that these regions do not play an important role in NMR conditioning.

In other studies, anterior lobe lesions in previously conditioned rabbits abolished the long-latency, adaptively timed component of the CS-elicited eyeblink response (NM responses were not studied) and replaced it with a short-latency, short-duration response that could not be extinguished (Perrett et al., 1993; Perrett and Mauk, 1995). In related studies, blockade of GABAergic Purkinje cell inputs to the cerebellar nuclei with nuclear inf usions of picrotoxin produced similar short-latency CS-elicited responses, although now with a longer plateau phase extending through the CS period (Garcia and Mauk, 1998; Ohyama and Mauk, 2001). It has been suggested that these studies reveal learning-related plasticities at the cerebellar nuclear and cerebellar cortical levels. At the nuclear level, CS-related mossy fiber collateral inputs may be potentiated, whereas at the cortical level, the CS-US interval is learned so as to provide an accurately timed inhibition on the nuclei and thereby to produce a CR with appropriate topography (Medina and Mauk, 2000; Ohyama and Mauk, 2001).

Here, we see no evidence for short-latency, CS-driven NM 
responses after inactivation of lobule HVI or lobule IV/V. The main effect of CNQX inactivation of lobule HVI was to abolish CRs, but as the effects dissipated, CR latencies were mildly extended (Fig. 5). However, it should be remembered that shortlatency responses have been revealed by lesioning, or pharmacologically blocking, inhibition on the cerebellar nuclei. In contrast, we blocked AMPA receptor-mediated transmission within the cortex so that intrinsic properties of Purkinje cells would maintain some inhibitory drive to the cerebellar nuclei. Our procedures would produce less disinhibition on the cerebellar nuclei, so the absence of short-latency, CS-driven responses does not rule out the possibility of plasticity at the cerebellar nuclei. The suggestion that cortical and nuclear plasticities combine to mediate eyeblink/ NMR conditioning remains an attractive one, because it explains how the timed, inhibitory cerebellar cortical output might control increased excitability in the nuclei to generate a learned movement.

Our findings do differ sharply, however, from others described above in that the region of cerebellar cortex critical for acquisition and performance involves the eyeblink control regions of lobule HVI and does not involve the anterior lobe. These locations in lobule HVI are consistent with those in our earlier lesion and inactivation studies and with electrophysiological mapping (Yeo and Hesslow, 1998). Further studies will be needed to reveal whether there are memory consolidation processes within HVI and what aspects of this motor memory might be encoded.

\section{REFERENCES}

Albus J (1971) A theory of cerebellar function. Math Biosci 10:25-61. Andersson G, Hesslow G (1987) Inferior olive excitability after high frequency climbing fibre activation in the cat. Exp Brain Res 67:523-532.

Andersson G, Garwicz M, Hesslow G (1988) Evidence for a GABAmediated cerebellar inhibition of the inferior olive in the cat. Exp Brain Res 72:450-456.

Attwell PJ, Rahman S, Ivarsson M, Yeo CH (1999) Cerebellar cortical AMPA-kainate receptor blockade prevents performance of classically conditioned nictitating membrane responses. J Neurosci 19:RC45.

Colin P, Manil J, Desclin JC (1980) The olivocerebellar system. 1. Delayed and slow inhibitory effects: an overlooked salient feature of cerebellar climbing fibers. Brain Res 187:3-27.

Garcia KS, Mauk MD (1998) Pharmacological analysis of cerebellar contributions to the timing and expression of conditioned eyelid responses. Neuropharmacology 37:471-480.

Gilbert P (1974) A theory of memory that explains the function and structure of the cerebellum. Brain Res 70:1-18.

Gilbert P (1975) How the cerebellum could memorize movements. Nature 254:688-689.

Gormezano I, Gibbs CM (1988) Transduction of the rabbit's nictitating membrane response. Behav Res Methods 20:18-21.

Gormezano I, Schneiderman N, Deaux EG, Fuentes I (1962) Nictitating membrane: classical conditioning and extinction in the albino rabbit. Science 138:33-34.

Gruart A, Yeo CH (1995) Cerebellar cortex and eyeblink conditioning: bilateral regulation of conditioned responses. Exp Brain Res 104:431-448.

Hardiman MJ, Yeo CH (1992) The effect of kainic acid lesions of the cerebellar cortex on the conditioned nictitating membrane response in the rabbit. Eur J Neurosci 4:966-980.

Hardiman MJ, Ramnani N, Yeo CH (1996) Reversible inactivations of the cerebellum with muscimol prevent the acquisition and extinction of conditioned nictitating membrane responses in the rabbit. Exp Brain Res 110:235-247.

Hesslow G (1994a) Correspondence between climbing fibre input and motor output in eyeblink-related areas in cat cerebellar cortex. J Physiol (Lond) 476:229-244.

Hesslow G (1994b) Inhibition of classically conditioned eyeblink responses by stimulation of the cerebellar cortex in the decerebrate cat. J Physiol (Lond) 476:245-256.

Hesslow G (1995) Classical conditioning of eyeblink in decerebrate cats and ferrets. In: Neural control of movement (Ferrell WR, Proske U, eds), pp 117-122. New York: Plenum.

Hesslow G, Ivarsson M (1994) Suppression of cerebellar Purkinje cells during conditioned responses in ferrets. NeuroReport 5:649-652.

Hesslow G, Svensson P, Ivarsson M (1999) Learned movements elicited by direct stimulation of cerebellar mossy fiber afferents. Neuron 24:179-185.

Ito M (1982) Cerebellar control of the vestibulo-ocular reflex-around the flocculus hypothesis. Annu Rev Neurosci 5:275-296.

Ito M (1998) Cerebellar learning in the vestibulo-ocular reflex. Trends Cogn Sci 2:313-321.

Kim JJ, Thompson RF (1997) Cerebellar circuits and synaptic mechanisms involved in classical eyeblink conditioning. Trends Neurosci 20:177-181.

Krupa DJ, Thompson RF (1995) Inactivation of the superior cerebellar peduncle blocks expression but not acquisition of the rabbit's classically conditioned eye-blink response. Proc Natl Acad Sci USA 92:5097-5101.

Krupa DJ, Thompson JK, Thompson RF (1993) Localization of a memory trace in the mammalian brain. Science 260:989-991.

Marr D (1969) A theory of cerebellar cortex. J Physiol (Lond) 202:437-470.

Mauk MD, Thompson RF (1987) Retention of classically conditioned eyelid responses following acute decerebration. Brain Res 403:89-95.

Medina JF, Mauk MD (2000) Computer simulation of cerebellar information processing. Nat Neurosci [Suppl] 3:1205-1211.

Montarolo PG, Palestini M, Strata P (1982) The inhibitory effect of the olivocerebellar input on the cerebellar Purkinje cells in the rat. J Physiol (Lond) 332:187-202.

Nordholm AF, Thompson JK, Dersarkissian C, Thompson RF (1993) Lidocaine infusion in a critical region of cerebellum completely prevents learning of the conditioned eyeblink response. Behav Neurosci 107:882-886.

Ohyama T, Mauk MD (2001) Latent acquisition of timed responses in cerebellar cortex. J Neurosci 21:682-690.

Perrett SP, Mauk MD (1995) Extinction of conditioned eyelid responses requires the anterior lobe of cerebellar cortex. J Neurosci 15:2074-2080.

Perrett SP, Ruiz BP, Mauk MD (1993) Cerebellar cortex lesions disrupt learning-dependent timing of conditioned eyelid responses. J Neurosci 13:1708-1718.

Ramnani N, Yeo CH (1996) Reversible inactivations of the cerebellum prevent the extinction of conditioned nictitating membrane responses in rabbits. J Physiol (Lond) 495:159-168.

Raymond JL, Lisberger SG, Mauk MD (1996) The cerebellum: a neuronal learning machine? Science 272:1126-1131.

Welsh JP, Harvey JA (1998) Acute inactivation of the inferior olive blocks associative learning. Eur J Neurosci 10:3321-3332.

Yeo CH, Hardiman MJ (1992) Cerebellar cortex and eyeblink conditioning: a reexamination. Exp Brain Res 88:623-638.

Yeo CH, Hesslow G (1998) Cerebellum and conditioned reflexes. Trends Cogn Sci 2:322-330.

Yeo CH, Hardiman MJ, Glickstein M (1984) Discrete lesions of the cerebellar cortex abolish the classically conditioned nictitating membrane response of the rabbit. Behav Brain Res 13:261-266.

Yeo CH, Hardiman MJ, Glickstein M (1985a) Classical conditioning of the nictitating membrane response of the rabbit. II. Lesions of the cerebellar cortex. Exp Brain Res 60:99-113.

Yeo CH, Hardiman MJ, Glickstein M (1985b) Classical conditioning of the nictitating membrane response of the rabbit. III. Connections of cerebellar lobule HVI. Exp Brain Res 60:114-126.

Yeo CH, Lobo DH, Baum A (1997) Acquisition of a new-latency conditioned nictitating membrane response-major, but not complete, dependence on the ipsilateral cerebellum. Learn Mem 3:557-577. 